\title{
EEG Oscillations Are Modulated in Different Behavior- Related Networks during Rhythmic Finger Movements
}

\author{
@Martin Seeber, Reinhold Scherer, and @Gernot R. Müller-Putz \\ Graz University of Technology, Institute of Neural Engineering, Laboratory of Brain- Computer Interfaces Graz, and BioTechMed-Graz, Graz 8010, Austria
}

\begin{abstract}
Sequencing and timing of body movements are essential to perform motoric tasks. In this study, we investigate the temporal relation between cortical oscillations and human motor behavior (i.e., rhythmic finger movements). High-density EEG recordings were used for source imaging based on individual anatomy. We separated sustained and movement phase-related EEG source amplitudes based on the actual finger movements recorded by a data glove. Sustained amplitude modulations in the contralateral hand area show decrease for $\alpha(10-12 \mathrm{~Hz})$ and $\beta(18-24 \mathrm{~Hz})$, but increase for high $\gamma(60-80 \mathrm{~Hz})$ frequencies during the entire movement period. Additionally, we found movement phase-related amplitudes, which resembled the flexion and extension sequence of the fingers. Especially for faster movement cadences, movement phase-related amplitudes included high $\beta(24-30 \mathrm{~Hz})$ frequencies in prefrontal areas. Interestingly, the spectral profiles and source patterns of movement phase-related amplitudes differed from sustained activities, suggesting that they represent different frequency-specific large-scale networks. First, networks were signified by the sustained element, which statically modulate their synchrony levels during continuous movements. These networks may upregulate neuronal excitability in brain regions specific to the limb, in this study the right hand area. Second, movement phase-related networks, which modulate their synchrony in relation to the movement sequence. We suggest that these frequency-specific networks are associated with distinct functions, including top-down control, sensorimotor prediction, and integration. The separation of different large-scale networks, we applied in this work, improves the interpretation of EEG sources in relation to human motor behavior.
\end{abstract}

Key words: EEG source imaging; finger movements; large-scale networks; neural oscillations; sensorimotor system; spectral profiles

Significance Statement

EEG recordings provide high temporal resolution suitable to relate cortical oscillations to actual movements. Investigating EEG sources during rhythmic finger movements, we distinguish sustained from movement phase-related amplitude modulations. We separate these two EEG source elements motivated by our previous findings in gait. Here, we found two types of large-scale networks, representing the right fingers in distinction from the time sequence of the movements. These findings suggest that EEG source amplitudes reconstructed in a cortical patch are the superposition of these simultaneously present network activities. Separating these frequency-specific networks is relevant for studying function and possible dysfunction of the cortical sensorimotor system in humans as well as to provide more advanced features for brain-computer interfaces.

\section{Introduction}

Generation of motoric actions for interacting appropriately with the environment is one of the key functions of the human brain. Sequencing of our body movements is essential for performing motoric tasks. Significant progress was achieved in studying relationships between brain activity and rhythmic motor behavior

Received May 30, 2016; revised July 28, 2016; accepted Sept. 10, 2016.

Author contributions: M.S., R.S., and G.R.M.-P. designed research; M.S. performed research; M.S. contributed unpublished reagents/analytic tools; M.S. analyzed data; M.S., R.S., and G.R.M.-P. wrote the paper.

This work was supported by the Land Steiermark project rE(EG)map! and BioTechMed Graz.

The authors declare no competing financial interests.

Correspondence should be addressed to either Dr. Martin Seeber or Dr. Gernot R. Müller Putz, Graz University of

Technology, Stremayrgasse 16, Graz 8010, Austria. E-mail: seeber@tugraz.at or gernot.mueller@tugraz.at.

DOI:10.1523/JNEUROSCI.1739-16.2016

Copyright $\odot 2016$ the authors $\quad 0270-6474 / 16 / 3611671-11 \$ 15.00 / 0$ in humans (Gerloff et al., 1998; Toma et al., 2002; Pollok et al., 2005; Miller et al., 2009; Houweling et al., 2010; Hermes et al., 2012). Yet, the temporal relation between cortical activities in different brain areas and human motor behavior is not fully understood. In this study, we investigate EEG source oscillations in various brain regions during rhythmic finger movements. Because of the high temporal resolution of EEG source reconstructed signals (Baillet et al., 2001; Michel et al., 2004), it is possible to investigate the temporal relation of cortical activities to movement sequences.

During movements, $\alpha$ and $\beta$ oscillations are suppressed in sensorimotor areas, but high $\gamma$ amplitudes are increased compared with a premovement reference period (Pfurtscheller and Aranibar, 1977; Pfurtscheller et al., 1997; Crone et al., 1998a, b; Müller et al., 2003; Miller et al., 2007; Scherer et al., 2009; Hermes 
et al., 2012). These relative amplitude decreases and increases were suggested to represent altered synchrony states (eventrelated (de)synchronization [ERD/ERS]) of underlying neuronal populations (for review, see Pfurtscheller and Lopes da Silva, 1999; Neuper and Pfurtscheller, 2001). Especially, $\beta$ oscillations were discussed to play a prominent role in motor function (for review, see Engel and Fries, 2010; Jenkinson and Brown, 2011). For faster movement rates, $\beta$ ERD becomes stronger (Houweling et al., 2010; Yuan et al., 2010), and its amplitude does not return to premovement levels anymore during finger tapping (Yuan et al., 2010; Hermes et al., 2012). Additionally, $\beta$ amplitudes were found to decrease prior movement onset and increase afterward, forming a comodulated pattern with the time sequence of rhythmic finger movements (Toma et al., 2002; Houweling et al., 2010). In sensorimotor regions, $\beta$ ERD was suggested to represent an increased excitability state of neuronal populations, whereas $\beta$ ERS was interpreted as active inhibition of neuronal circuitry (for review, see Neuper and Pfurtscheller, 2001). In contrast to $\beta$ oscillations, high $\gamma$ amplitudes increase prior movement onsets, and its time course resembles the movement sequence during finger tapping (Miller et al., 2009; Hermes et al., 2012).

In recent walking experiments of our group (Wagner et al., 2012, 2014; Seeber et al., 2014, 2015), we report two different EEG source elements. First, sustained $\beta$ ERD along with high $\gamma$ increase, which are present during the entire gait cycle. We interpreted this pattern as altered synchrony states in respective central sensorimotor networks. Second, we found gait cyclerelated amplitude modulations in the high $\beta$ /low $\gamma$ and high $\gamma$ frequency range, which were conversely modulated to each other. Interestingly, the spectral profiles of sustained ERD/ERS did not match those of gait cycle-related amplitude modulations (Seeber et al., 2014, 2015). Different spectral profiles were suggested to represent different large-scale neuronal network interactions (Donner et al., 2011; Siegel et al., 2012). Therefore, we hypothesize that, during continuous rhythmic movements, one type of large-scale networks (up)regulate the neuronal excitability state in specific cortical regions, but other networks represent the temporal structure of the movement sequence. In this work, we test this viewpoint for rhythmic finger movements to derive principles, which may unify findings from both extremities. In contrast to foot movements, the fingers are represented laterally in the somatotopic arrangement of the motor cortex (Jasper and Penfield, 1949). Because of the low spatial resolution of the EEG, it was not possible to unambiguously separate supplementary motor area (SMA) from central motor areas in the walking studies. Because of the lateral representation of the fingers, we further hypothesize in this work that sustained ERD/ERS and movement phase-related sources are different in both respect, their spectral profile, and, additionally, in their spatial patterns during rhythmic finger movements.

\section{Materials and Methods}

Experiment and recordings. We recorded EEG from 18 right-handed healthy volunteers ( $24 \pm 4$ years, 9 female) during rhythmic right finger movements. Each participant gave informed consent to the study, which was performed in accordance to the Declaration of Helsinki. During the experiments, subjects were seated in a comfortable chair in an electrical shielded room with their right hand supinated on an armrest. A blue dot was shown in the center of a computer screen and was blinking three times, indicating either 0.67 or $1.5 \mathrm{~Hz}$ finger movement cadences (see Fig. 1a). Participants were asked to rhythmically open and close their right hand continuously, imitating the previously displayed blinking cadence of the dot. Subjects initiated their movements after the dot stopped blinking. Importantly, no external stimuli were present during the movement period. Because we aimed to study the cortical motor system, we minimized the impact from other sensory modalities. A movement trial ended when the dot disappeared from screen. In total, one trial lasted $13 \mathrm{~s}$ and was followed by a 1-2 s random break with a black screen. Ninety trials were completed for both movement cadences, respectively. Participants were asked to fixate the dot with their eyes, not to count during the movements and avoid any additional movement as good as possible. At the beginning of each trial, a red arrow was additionally displayed. It was pointing down to indicate finger movements without the thumb or pointing toward left for thumb movements only. The movement experiment was recorded in three blocks. Before and after each movement block, we recorded EEG during a resting period lasting 3 min each. During the resting period, the blue dot was constantly shown on the screen for gaze fixation. We pooled finger and thumb movement data for each tapping cadence because we investigate temporal patterns of movement sequences in this work, not which fingers were moved.

EEG was recorded combining five 16 channel amplifiers (g.USBamp, g.tec) sampled to $512 \mathrm{~Hz}$ and bandpass filtered between $0.1 \mathrm{~Hz}$ and $200 \mathrm{~Hz}$. A notch filter was set to suppress $50 \mathrm{~Hz}$ line noise. Passive $\mathrm{Ag} / \mathrm{AgCl}$ electrodes were mounted at selected 72 positions (see Fig. $1 d$ ) of the 5\% international 10/20 EEG system (Oostenveld and Praamstra, 2001). Reference and ground electrodes were placed on the left and right mastoid, respectively. Electrode impedances were kept $<5 \mathrm{k} \Omega$. Electrode positions were measured using a 3D localizer (Elpos, Zebris). Finger movements were digitalized using a data glove (5DT Data Glove 14 Utra, 5DT). Structural MRI T1 scans $(\mathrm{TE}=2.07 \mathrm{~ms}$, TR $=1560 \mathrm{~ms}$, voxel size $=1 \times 1 \times 1 \mathrm{~mm}, 3$ tesla Skyra, Siemens) were acquired in a postscreening session for each individual brain.

EEG preprocessing. EEG was visually inspected for prominent artifacts, and related time intervals were excluded from further analysis $(94.1 \%$ were kept). Subsequent independent component analysis (ICA) (Hyvärinen, 1999) (FastICA, https://research.ics.aalto.fi/ica/fastica/) was used to identify noncortical components. Artifact stereotypical ICs were selected based on their time course, power spectra, and topographies and were rejected ranging from 3 to 7 ICs per subject. After ICA correction, signal periods exceeding amplitudes of $\pm 200 \mu \mathrm{V}$ were detected and ignored for subsequent analysis. Considering the previously excluded time periods, $88.3 \%$ of the raw data points remained in total for further analysis. Subsequently, EEG recordings were rereferenced to common average reference.

EEG source imaging. EEG source imaging (Baillet et al., 2001; Darvas et al., 2004; Michel et al., 2004; Michel and Murray, 2012) was applied based on realistic head models using individual MRI scans. To describe the propagation of electrical fields from the cortical surface to the scalp, a forward model was formulated as boundary element model (BEM) for each subject using OpenMEEG (Kybic et al., 2005; Gramfort et al., 2010). The BEM consisted of four surface layers (brain, inner skull, outer skull, head surface), which were reconstructed from individual T1 MRI scans. Cortical reconstruction and volumetric segmentation were performed with the FreeSurfer image analysis suite (Dale et al., 1999; Fischl, 2012) (http://surfer.nmr.mgh.harvard.edu/). BEM and electrode positions were coregistered, matching four anatomical landmarks (nasion, vertex, left and right preauricular points). EEG sources were modeled perpendicular to individual gray matter surfaces and consisted of 5000 vertices. Resting EEG periods were used to calculate the noise covariance matrix for whitening the lead field matrix. For solving the inverse problem, sLORETA (Pascual-Marqui, 2002) implemented in the Brainstorm toolbox (Tadel et al., 2011) (http://neuroimage.usc.edu/brainstorm) was applied. sLORETA is a standardized variant of the minimum norm inverse solution (Hämäläinen and Ilmoniemi, 1994). To prevent overfitting, no EEG data of the movement trials and no frequency-specific information was used to calculate the inverse Kernel.

EEG source amplitude envelope (AE) analysis and glove data alignment. AEs were computed in the EEG source space using complex Morlet wavelet (Morlet et al., 1982) for time-frequency (TF) decomposition (2-100 Hz, $2 \mathrm{~Hz}$ steps). Parameters for the mother wavelet were set to FWHM of $3 \mathrm{~s}$ for the Gaussian kernel at a center frequency of $1 \mathrm{~Hz}$. 
a
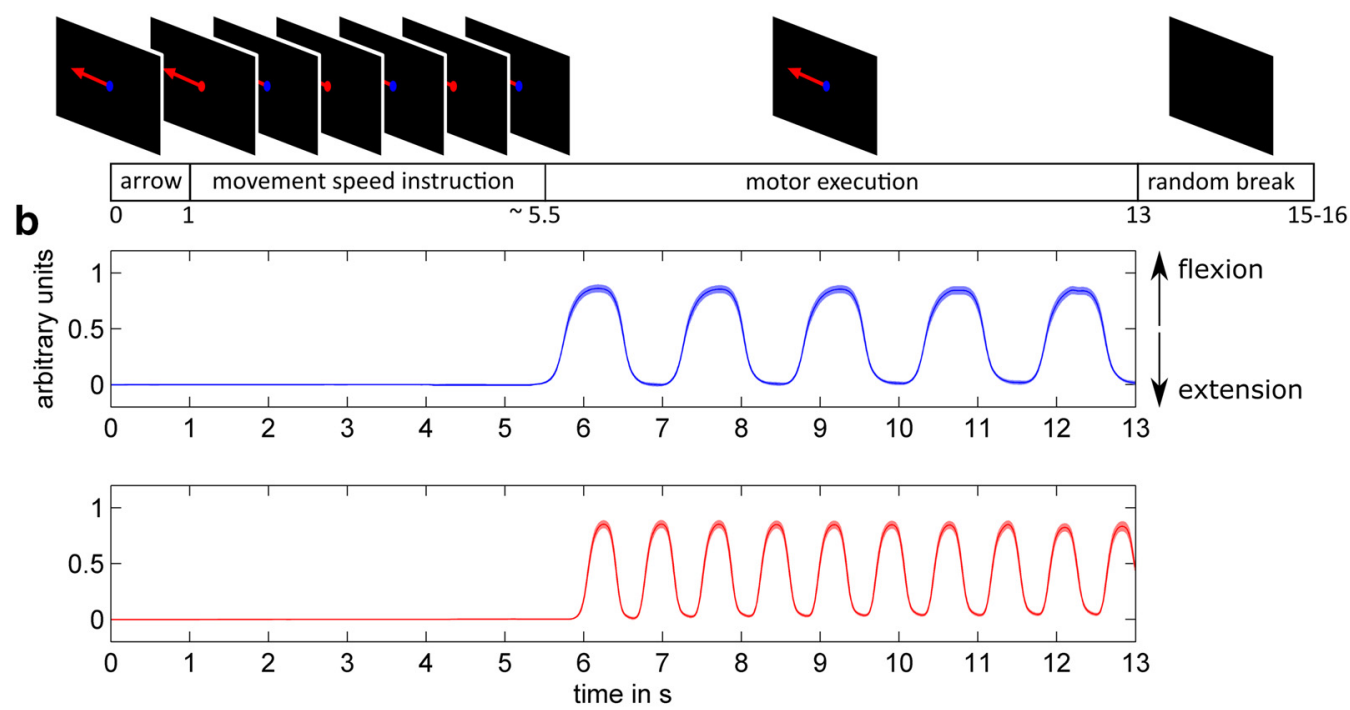

C
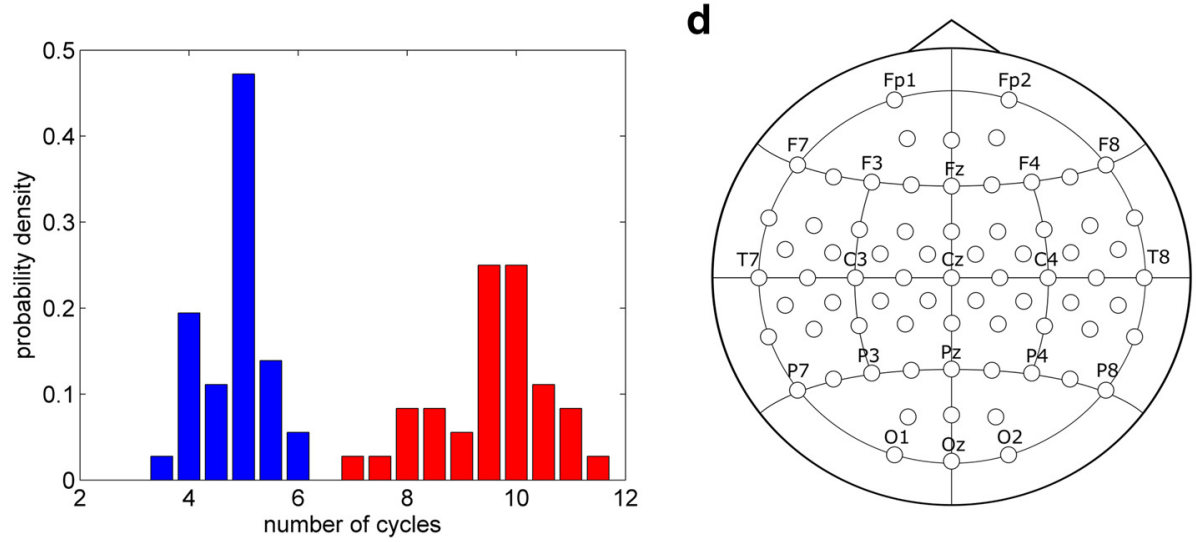

Figure 1. Task, behavior, and EEG montage. $\boldsymbol{a}$, Time line of the movement task instruction displayed on the computer screen. $\boldsymbol{b}$, Glove data (mean \pm SEM) after alignment of trials for the slow (blue) and fast (red) tapping cadence. $\boldsymbol{c}$, Histogram of performed movement cycles. $\boldsymbol{d}$, EEG montage. Circles represent electrodes.

Magnitudes of TF decomposed signals were calculated to obtain EEG source amplitude envelopes.

Glove data were used to align and warp the EEG source AE. The first principal component (Jolliffe, 2002) of the glove sensors was calculated to get a single curve describing the finger movements. Movement cycles were quantized in quarters, which were determined by the turning points and zeniths of this movement curve for each trial. Subsequently, the glove data were stretched and bended to match these sampling points and the average movement cycle quantity for all trials of the two different movement cadences, respectively. The glove data were equally used to realign and time warp the EEG source $\mathrm{AE}$ on a single-trial basis.

EEG source amplitudes were normalized relative to a nonmovement reference period (second 2-4 of the trial) in decibels $(\mathrm{dB})$ for each subject. To improve signal quality for higher frequencies $(>30 \mathrm{~Hz})$, we suppressed broadband source space activity, which we consider to reflect electromuscular activity during movement tasks, using a spectral decomposition approach (Seeber et al., 2015). These AEs then were low and high pass filtered at $0.3 \mathrm{~Hz}$, respectively, to separate sustained and dynamic AEs (see Fig. 2). This frequency is a trade-off between capturing sustained effects precisely without affecting modulations in the movement frequency range. Sustained $\mathrm{AE}$ in our analysis corresponds to sustained ERD/ERS (Pfurtscheller and Lopes da Silva, 1999) measures using a logarithmic scale in $\mathrm{dB}$. To assess movement phase-related amplitudes (MPAs), we investigated the dynamic AE in relation to the movement cycle phase from the glove data. To do so, we quantized the movement cycle phase into $4^{\circ}(\pi / 45)$ steps. TF source amplitude values were averaged for each phase step to determine amplitude curves as function of the phase in the movement cycle. To quantify this relationship, we computed the modulation (analytical) amplitude of the resulting MPA curves using Hilbert transform.

These calculations were processed for every source vertex and analyzed frequency resulting in source images for both, sustained and MPA.

In this work, we present time courses, frequency spectra, and TF plots in a left and right ROI. These ROIs were determined based on the centers of the well-known $\beta$ (18-24 Hz) ERD patterns and were subsequently normalized to the same spatial size. Frequency ranges for the topographical analysis were chosen based on peaks in the frequency spectra. We also investigated amplitude comodulation between cortical areas using the ROIs as seed regions. Amplitude comodulation was investigated because it was previously shown to reveal similar connectivity patterns as fMRI networks (Brookes et al., 2011; Engel et at., 2013). To ignore volume conduction effects, we computed the imaginary part of coherence (Nolte et al., 2004) between AEs.

Group analyses were enabled by aligning and projecting individual cortices to match the ICBM 152 surface (Fischl et al., 1999; Tadel et al., 2011). Significant clusters in EEG source images were determined and corrected for multiple comparisons using nonparametric permutation tests (Nichols and Holmes, 2002; Maris and Oostenveld, 2007). In detail, EEG source amplitudes of randomly selected halves ( 9 of 18) subjects were sign flipped and averaged to obtain a random distribution. We performed $10^{4}$ permutations for each source topography. From these analysis, we applied a primary threshold of $p=1 \mathrm{e}-3$ for the ERD/ERS and MPA images, except for high $\gamma$ MPA images. For these images, $p=0.01$ was used due to the lower signal-to-noise ratio for higher frequencies. 
After applying the primary thresholds, the resulting clusters were compared with maximum clusters sizes from again $10^{4}$ permutations. We chose the same significance levels as described above for these cluster-level statistics. Significant amplitude comodulations were determined using the same procedure for the $\mathrm{AE}$ coherence.

\section{Results}

\section{Behavioral data}

Glove data showed that subjects performed 5 and 9.75 movement cycles on average for the slow and fast movement cadence, respectively (Fig. 1b,c). These data also show that the aligning and warping procedure was successful due to the small standard error of the mean (SEM). Considering the movement duration, the performed movement cadences were 0.66 and $1.37 \mathrm{~Hz}$. The visual instruction (i.e., dot blinking frequency) were $0.67 \mathrm{~Hz}$ and $1.5 \mathrm{~Hz}$, so the movement frequency for the faster movement was not exactly as instructed. However, the histogram of performed movements shows two clear, nonoverlapping groups for the slow and fast cadence (Fig. 1c).

\section{Sustained and movement phase-related AE}

We separated source AE in two elements: a sustained and a dynamic one. The latter, we hypothesized to represent the movement phases. These two elements are significantly present in sensorimotor areas for both movement cadences (Figs. 2, $3 b-d, 4 b-d)$. First, for the sustained AE element, amplitudes decrease in the $\alpha(10-12 \mathrm{~Hz})$ and $\beta 1$ $(18-24 \mathrm{~Hz})$, but increase in the high $\gamma(60-80 \mathrm{~Hz})$ frequency range during movements. This amplitude de/increase starts $2.5 \mathrm{~s}$ and $2.6 \mathrm{~s}$ before the slow and fast movement, respectively, peaks at its onset, and remains at a sustained level during the entire movement duration (Figs. 2, 3c, 4c). Second, the dynamic AE element shows a clear relationship to the movement phase in the $\beta 1(18-24 \mathrm{~Hz})$ and $\beta 2(24-30 \mathrm{~Hz})$ frequency range and resembled the glove data (Figs. 2, 3b,f, 4b,f). Movement phase-related $\beta 2$ amplitudes peak at the start of a movement cycle (phase: $-3.00 \mathrm{rad}$ and $-2.93 \mathrm{rad}$ ) and are lowest preceding maximal finger flexion at $-0.97 \mathrm{rad}$ and $-0.63 \mathrm{rad}$ during slow and fast movement cadences, respectively. For the slow movement cadence, a second peak is present at $0.70 \mathrm{rad}$ (Figs. $3 f, 4 f$ ). We also found that $\mathrm{AE}$ of different frequencies showed a similar temporal structure locked to the movement cycle but shifted to each other. $\beta 2$ precedes $\alpha$ amplitudes in the movement cycle. This delay was tested for significance $\left(\chi_{(1,17)}^{2}=21.33, p=3.9 \mathrm{e}-6\right)$ using a Friedman's test with factors frequency range $(n=2)$ and experimental condition $(n=2)$ in the right ROI, where $\beta 2$ and $\alpha$ MPA spatially overlap. Further, $\beta 2$ amplitudes are conversely modulated to high $\gamma$ amplitudes in a movement cycle. These amplitudes are negatively correlated to each other for slow (permutation test, $r=-0.90, p=1 \mathrm{e}-4$ ) and fast movements (permutation test, $r=-0.78, p=2 \mathrm{e}-4)$.
Interestingly, the MPA frequency spectra does not fully match with the spectra of sustained amplitude decreases/increases. Spectral $\beta$ peaks for the sustained decrease are at $20 \mathrm{~Hz}$ for both movement cadences. MPAs are additionally present in the $\beta 2$ frequency range, especially for the faster movement cadences (Figs. $3 g, 4 g$ ). Selecting these specific frequency ranges, we further analyzed the spatial patterns of both modalities.

\section{EEG source images}

The sources of the sustained AE element (Figs. 5a, 6a) show a well-known left hemisphere lateralized pattern in the right hand representation area of the sensorimotor cortex for $\beta 1$ desynchronization and high $\gamma$ increase. Apart from this contralateral hand area, we also located significant clusters in the homologous right sensorimotor area and paracentral regions for these frequency ranges. Sustained $\alpha$ amplitudes decrease less specific in bilateral sensorimotor areas. Friedman's test with factors frequency range $(n=2)$ and experimental condition $(n=2)$ determined a higher lateralization for $\beta 1$ compared with $\alpha$ desynchronization $\left(\chi_{(1,17)}^{2}=8.53, p=0.0035\right)$. Comparing fast with slow movement cadences, sustained $\beta 1$ ERD in the left cluster is significantly stronger during fast movements (Wilcoxon signed rank, $p=$ 0.0073 ). No significant difference in $\beta 1$ lateralization (Wilcoxon signed rank, $p=0.71$ ) was found.

In contrast to the well-known ERD/ERS patterns during movement, MPA sources revealed different sources (Figs. 5, 6). 
a

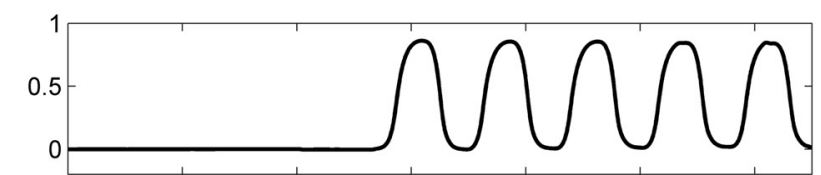

b
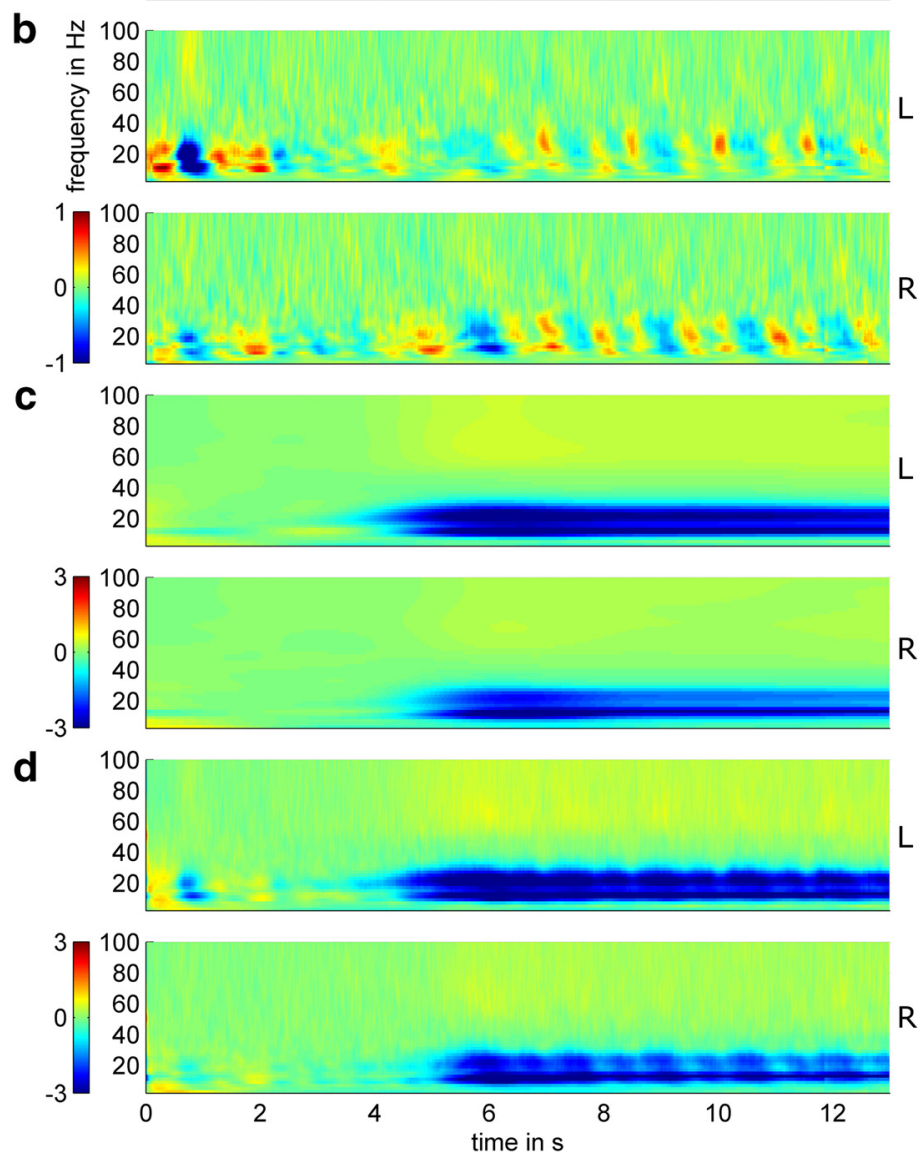

e
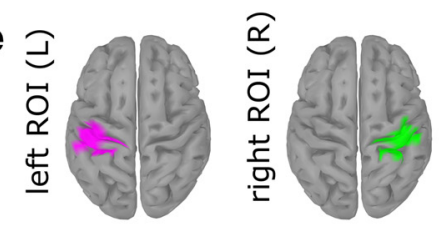

f 100
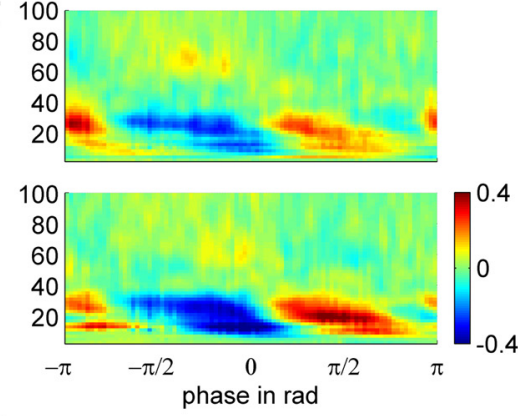

$\mathbf{g}$
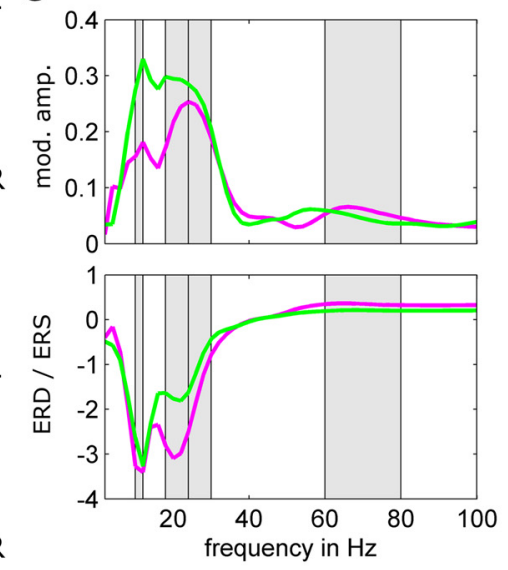

Figure 3. Dynamic and sustained AE in the right and left ROI during the slow movement cadence. $\boldsymbol{a}$, Glove data. $\boldsymbol{b}$ - $\boldsymbol{d}$, TF plot of dynamic (high pass filtered), sustained (low pass filtered), and original (unfiltered) source AE. $\boldsymbol{e}$, Right and left ROI. $\boldsymbol{f}$, Relation of dynamic AE to the movement phase, centered ( 0 rad) at peak displacement of the fingers. $\boldsymbol{g}$, Frequency spectra for sustained (bottom) and MPA (top) for the left/right ROI in magenta/green. Selected frequency ranges marked in gray shaded regions. Amplitudes in dB.

Because of the different spectral profiles we found in the sensorimotor regions, we pooled $\beta$ frequencies to compare the spatial patterns of both modalities in terms of lateralization. Friedman's test with factors modality (sustained ERD/ERS and MPA, $n=2$ ) and experimental condition $(n=2)$ identified different lateralization for $\beta$ frequencies $\left(\chi_{(1,17)}^{2}=22.53, p=2.1 \mathrm{e}-6\right) . \beta \mathrm{MPA}$ sources were localized to bilateral sensorimotor and paracentral regions. These activities are significantly lower (Wilcoxon signed rank, $p=2 \mathrm{e}-4$ ) during fast compared with slow movements. For the faster movement cadence, we additionally found MPA located in the right prefrontal cortex at $\beta 2$ frequencies (Fig. 6b). High $\gamma$ MPA sources were localized to the contralateral hand area and left paracentral region during slow movements. For fast movements, the paracentral cluster exclusively reached significance. $\alpha$ MPA sources showed a widespread pattern, which was strongest in right sensorimotor areas, but also covered bilateral superior parietal and paracentral regions (Figs. 5, 6).

\section{Amplitude comodulation between cortical areas}

We also observed well-pronounced amplitude modulations during the visual instruction strongest for $\alpha$ frequencies in the left ROI (Figs. $3 b, 4 b$ ). In this time interval, of course, there was no movement executed. To further investigate this modulation, we computed amplitude comodulation between the left ROI (seed region) to every other site of the cortex using $\alpha$ as carrier frequency. This analysis showed significant comodulation in visual areas for both movement conditions, which precede the left seed region (Fig. 7, left).

Furthermore, we focused on the analysis of $\beta 2$ MPA in the right ROI to test for significant comodulation with other cortical sites. During slow movements, subcortical areas are significantly comodulated with the right seed region and precedes in the movement cycle. For the fast cadence, prefrontal areas are significantly comodulated and are leading the right sensorimotor seed cluster (Fig. 7, right).

To provide further information about the temporal relation of $\beta 2$ MPA sources in different cortical areas, we rendered videos for both movement cadences showing $\beta 2$ amplitudes in dependence of the movement cycle phase (Movies 1,2). During slow movements, we observe two peaks during a movement cycle. The first peak occurs at the movement cycle onset at $-\pi / \pi$ and shows a pattern in bilaterally in sensorimotor hand areas. Subsequently, $\beta 2$ amplitudes decrease, reaching its minimum preceding the maximal displacement of the fingers (at $0 \mathrm{rad}$ ). Then a second 
a
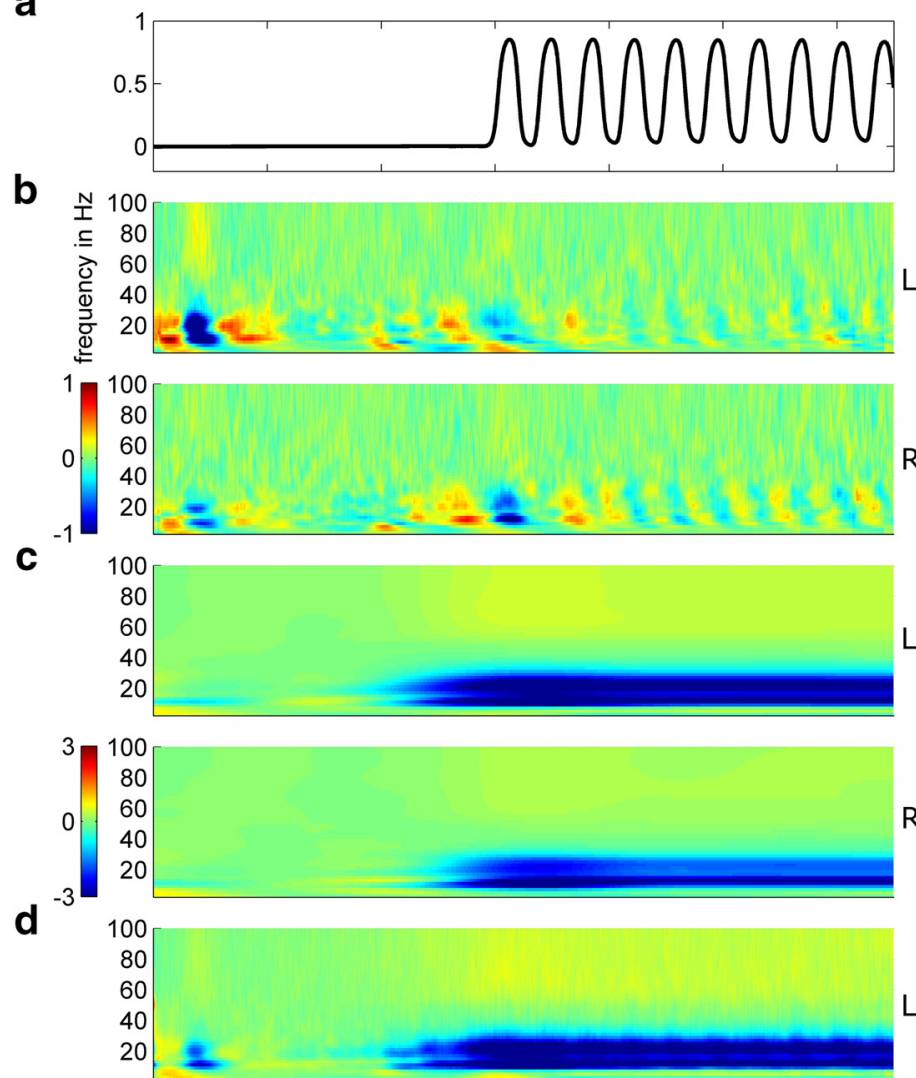

3
0
-3

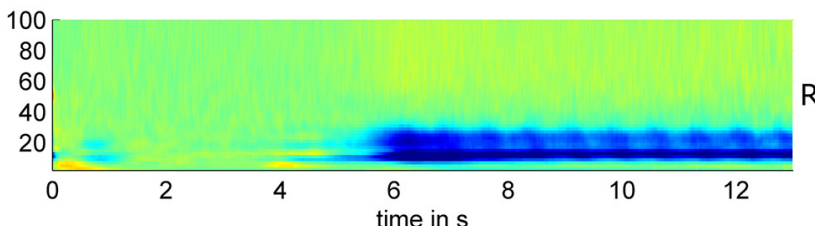

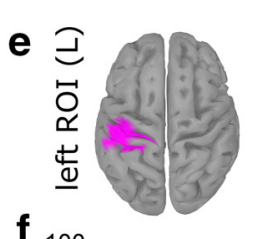

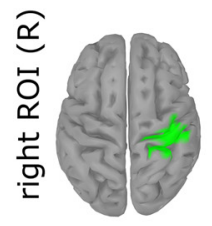

100

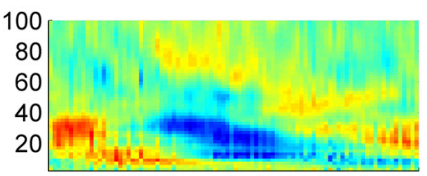

$\mathrm{R}$

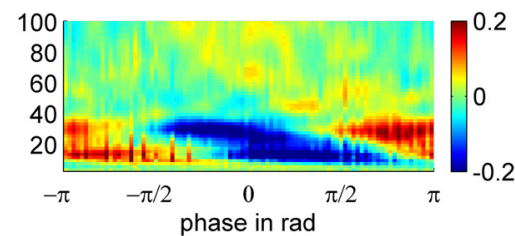

$\mathbf{g}$
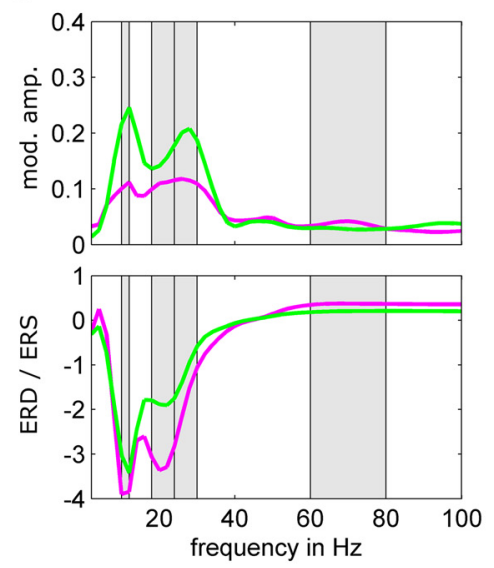

Figure 4. Dynamic and sustained AE in the right and left ROI during the fast movement cadence. $\boldsymbol{a}$, Glove data. $\boldsymbol{b}$ - $\boldsymbol{d}$, TF plot of dynamic (high pass filtered), sustained (low pass filtered), and original (unfiltered) source AE. e, Right and left ROI. $\boldsymbol{f}$, Relation of dynamic AE to the movement phase, centered ( 0 rad) at peak displacement of the fingers. $\boldsymbol{g}$, Frequency spectra for sustained (bottom) and MPA (top) for the left/right ROl in magenta/green. Selected frequency ranges marked in gray shaded regions. Amplitudes in $\mathrm{dB}$.

peak evolves, which is, compared with the first peak, rather associated with superior sensorimotor and central regions. During the fast movement cadence, the temporal sequence of $\beta 2$ amplitudes shares two characteristics with the slow movement cadence: that is, (1) the peak at the movement cycle onset, and (2) the $\beta 2$ minimum before the finger displacement maximum. Additionally, amplitudes in prefrontal areas are modulated in relation to the movement cycle. These prefrontal modulations show a different phase relation to the movement cycle, preceding amplitude envelopes in sensorimotor regions. In summary, one can observe that amplitudes start decrease and increase in frontal areas, which then spreads toward parietal areas while additional amplitude modulations in the sensorimotor network occur.

\section{Discussion}

In this study, we investigated EEG sources during rhythmic finger movements in humans. Analyzing these source we distinguish between sustained (de-)synchronization (ERD/ERS) and MPA modulations. We found that the sources of these two electrocortical elements are different, confirming our hypothesis.

The sustained ERD/ERS element replicated previous works showing $\alpha$ and $\beta$ desynchronization accompanied with high $\gamma$ increase in contralateral areas representing right finger movements (Pfurtscheller et al., 1997; Crone et al., 1998b; Miller et al., 2007). Further, the temporal properties of these features, evolving at $\sim 2 \mathrm{~s}$ before the movement are also in line with these studies. As well as that, $\alpha$ are less spatially specific than $\beta$ ERD sources (Crone et al., 1998a). Further, consistent with previous reports, we found sustained $\beta$ desynchronization (Hermes et al., 2012), which is getting stronger during faster rhythmic movements (Yuan et al., 2010).

$\alpha$ and $\beta$ desynchronization along with high $\gamma$ increase may signify a state of enhanced cortical excitability in associated areas, which facilitates motor processing. Previous studies indeed provide evidence for causal relationship of cortical oscillations and motor performance. These studies showed that transcranial alternating current stimulation at $\beta$ frequencies slows voluntary movements (Pogosyan et al., 2009), but stimulation at high $\gamma$ facilitates motor processing (Joundi et al., 2012). Consequently, $\beta$ and high $\gamma$ oscillations were found to possess opposing roles in the motor cortex, the former to inhibit, the latter to facilitate, dynamic motor actions during its preparation and performance. 
a
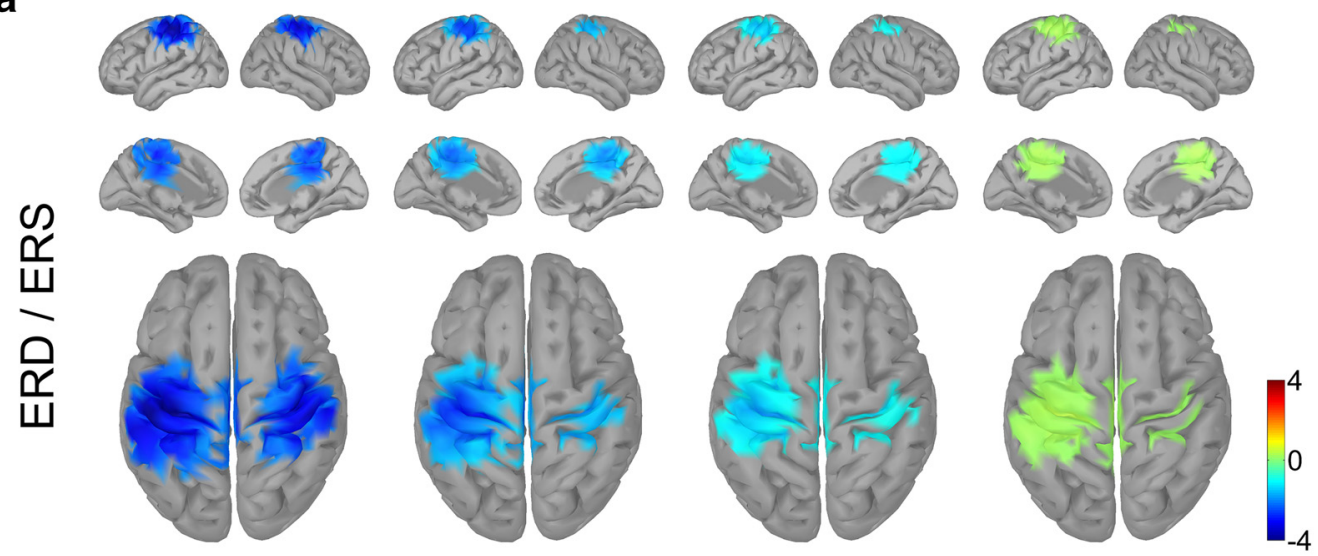

b
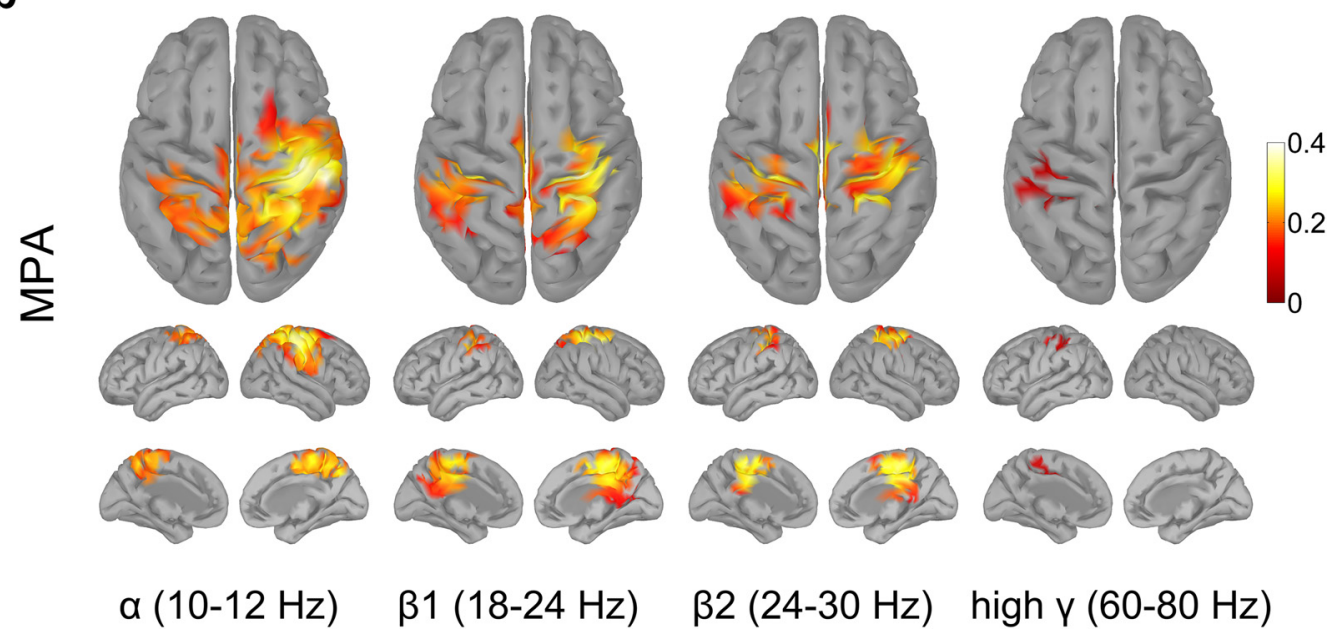

ß1 $(18-24 \mathrm{~Hz})$

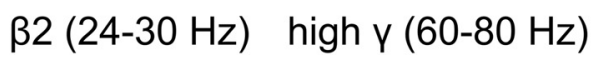

Figure 5. EEG source images of the slow movement cadence in selected frequency ranges (left to right column). $\boldsymbol{a}$, EEG sources of sustained ERD/ERS during movements are illustrated in blue/yellow-red. $\boldsymbol{b}$, EEG sources of movement MPAs are illustrated as modulation magnitude in red to white. Amplitudes in dB.

We found the MPA sources to be different from the wellknown ERD/ERS patterns during movement. EEG source imaging revealed different spatial patterns for these two elements (Figs. 5, 6). Further, the spectral profiles we investigated in left and right sensorimotor areas did not match. For instance, we found different $\beta$ peaks for the sustained ERD and MPA feature. Narrow-band peaks in the EEG frequency spectra reflect the summation of neuronal oscillations on a larger spatial scale. Neuronal oscillations mediate synchrony in neuronal populations forming frequency-specific networks (Buzsáki, 2006; Siegel et al., 2012). Because of spatiotemporal integration inherent in large-scale recordings as EEG, the amplitudes of these signals are markers of underlying network synchrony (Elul, 1971; Pfurtscheller and Lopes da Silva, 1999; Donner et al., 2011; Buzsáki et al., 2012). Amplitude modulation at a certain frequency, therefore, is a signature of modulated synchrony in a frequency-specific neuronal network. Based on this, we interpret sustained ERD/ERS and MPA to represent two different types of large-scale networks, due to the different spectral profiles and spatial patterns we report in this study.

First, networks represented by sustained ERD/ERS, which statically modulate their synchrony level during continuous movements. These patterns may upregulate neuronal excitability during motor preparation and performance specific to the limb, in this work the right hand area.
Second, MPAs are generated by other frequency-specific networks, which dynamically adapts their synchrony levels dependent on the phase in a movement cycle. Therefore, these networks provide information about the movement sequence timing. We found MPAs are well pronounced at $\beta$ frequencies in sensorimotor regions and SMA. This spatial pattern resembles the sensorimotor resting state network (Raichle, 2010). Notably, $\beta$ MPAs are significantly stronger during slow compared with fast movements, which was also reported previously (Toma et al., 2002; Houweling et al., 2010; Hermes et al., 2012). These studies further described $\beta$ amplitude modulations associated with different movements to merge at faster movements $(\geq 2 \mathrm{~Hz})$. We did not find this phenomenon probably because of the comparably lower movement cadences we studied. To build up neural synchrony, increasing time is needed for larger cortical populations (Buzsáki, 2006). This relationship could explain the larger MPA during the slower movement cadences, where longer time periods are available in movement cycle to build up synchronized networks.

Movement phase-related $\beta$ synchrony peaked at the start of a movement cycle, that is, at the initial position of the fingers, and it was diminished preceding the maximal fingers flexion (Fig. 4f). Because of the bilateral sensorimotor regions we found for $\beta$ MPA, it would be plausible that they also mediate sensorimotor processing between the hemispheres. The time course of $\beta$ modulation in the motor system was previously reported to be associated with predictive timing of upcoming external rhythmic visual (Saleh et al., 2010) 
a
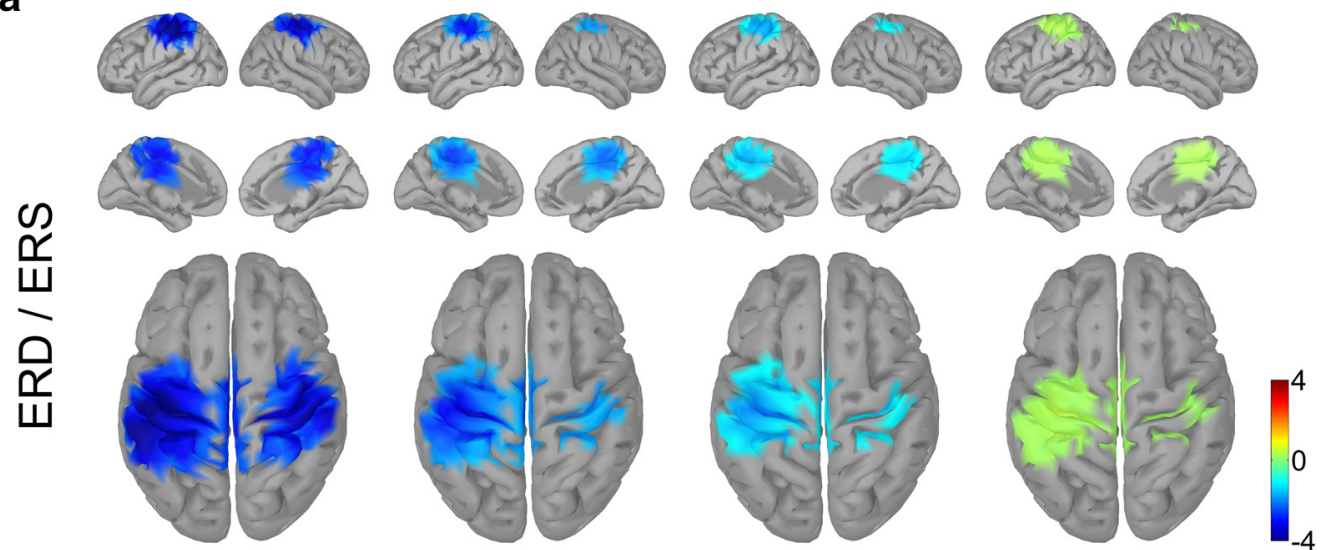

b
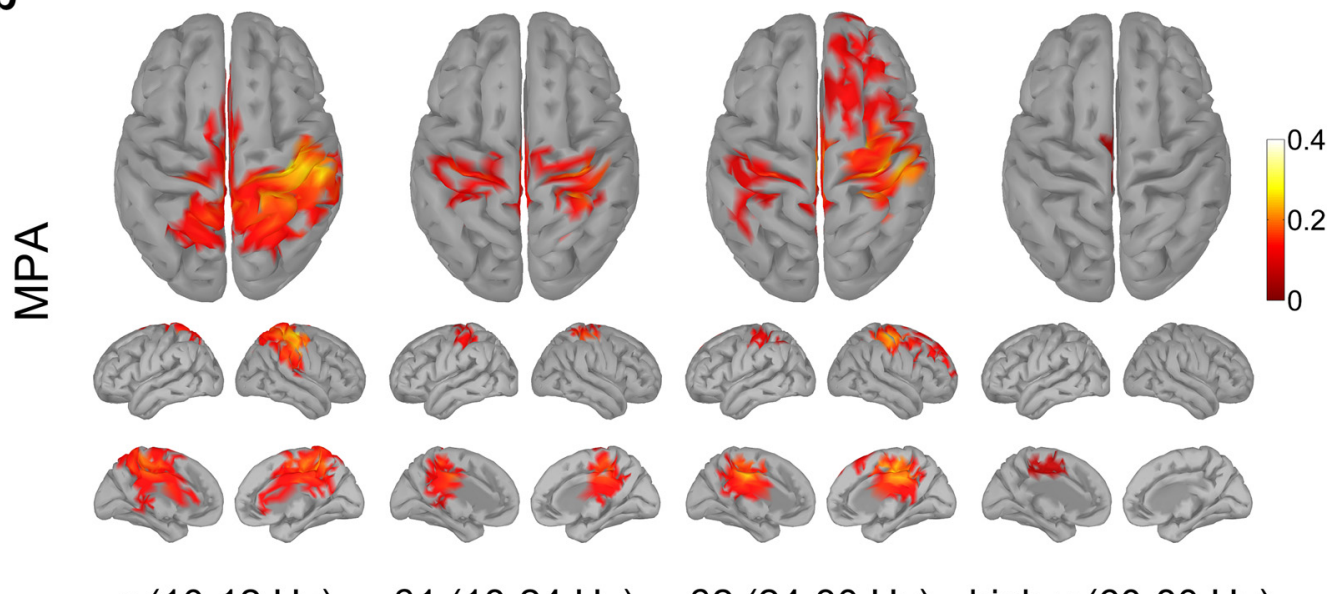

$\alpha(10-12 \mathrm{~Hz})$

$\beta 1(18-24 \mathrm{~Hz})$

$\beta 2(24-30 \mathrm{~Hz})$

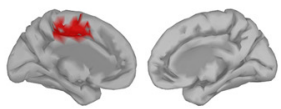

Figure 6. EEG source images of the fast movement cadence in selected frequency ranges (left to right column). $\boldsymbol{a}$, EEG sources of sustained ERD/ERS during movements are illustrated in blue/yellow-red. $\boldsymbol{b}$, EEG sources of movement MPAs are illustrated as modulation magnitude in red to white. Amplitudes in dB.

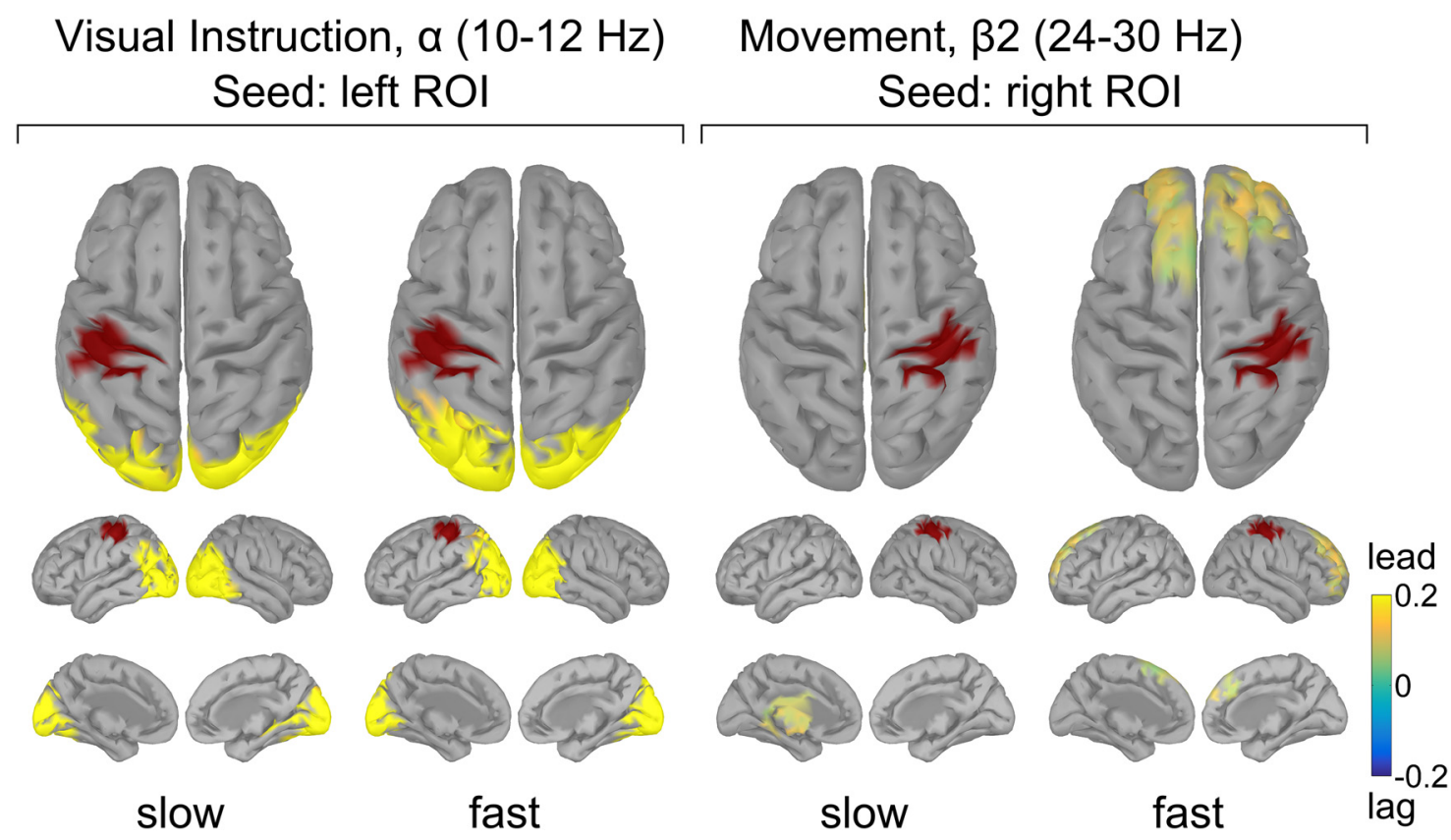

Figure 7. Amplitude comodulation between cortical areas. Left, Visual areas are comodulated with the left sensorimotor area (seed region in red) during visual instruction. Right, Subcortical regions are comodulated with the right sensorimotor area (seed region in red) during slow, but prefrontal areas during fast movement cadences. 


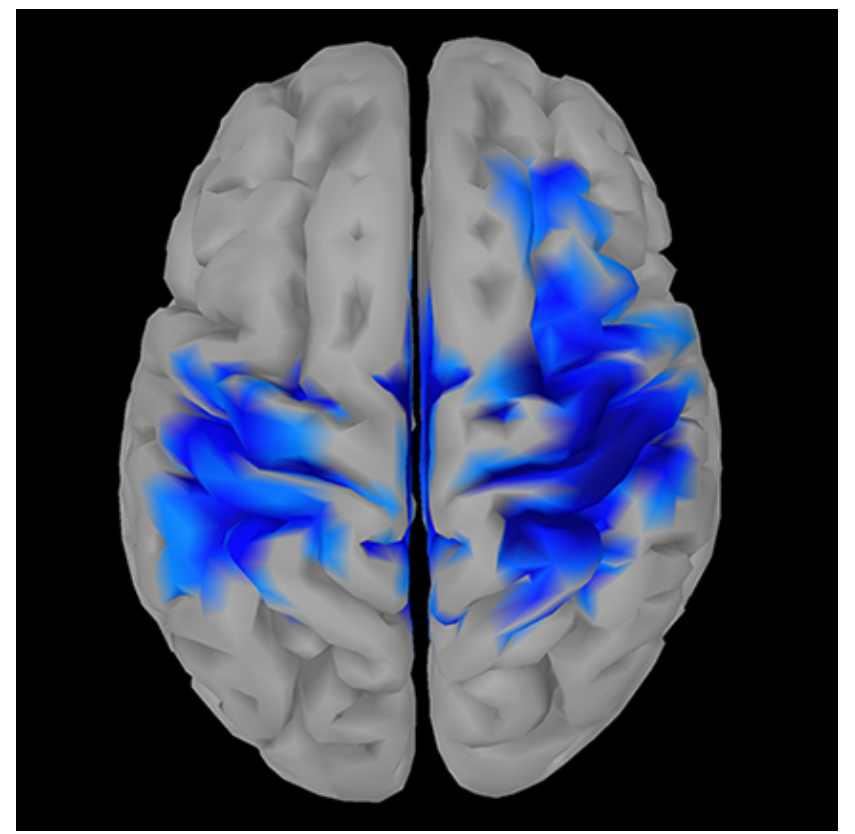

Movie 1. EEG source amplitudes for high $\beta(24-30 \mathrm{~Hz})$ frequencies during the slow movement cadence as function of the phase in a movement cycle. Phase axis in radian, ranging from $-\pi$ to $\pi$, is centered $(0)$ at the maximal flexion of the fingers. Relative amplitude decreases/increases are illustrated in blue/red. EEG source amplitude threshold was set to $50 \%$ of the color scale maximum; the minimum cluster size was set to 20 vertices.

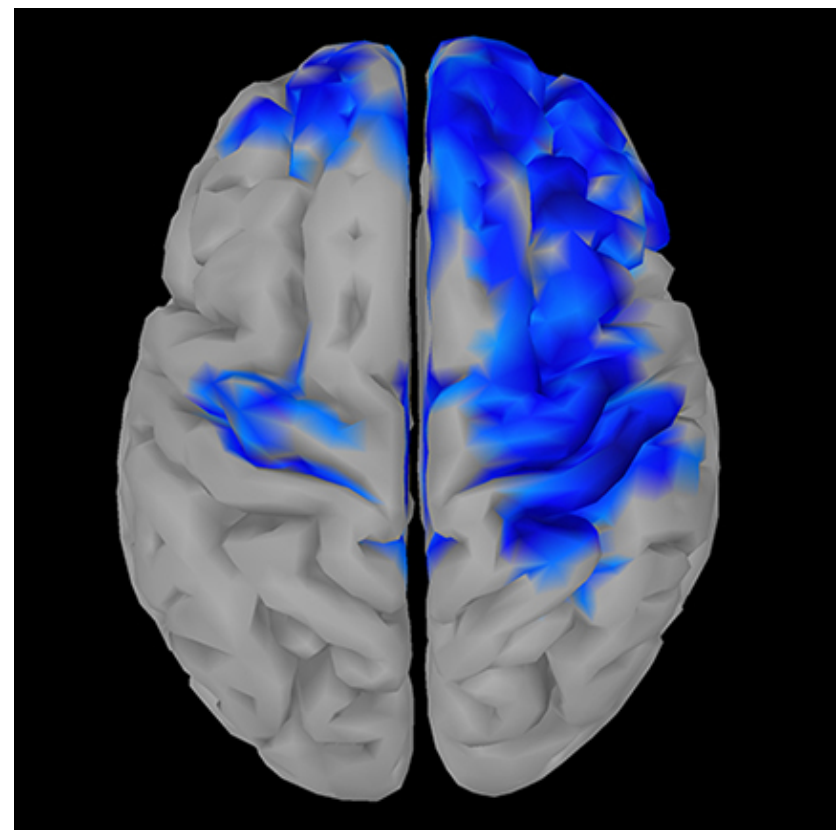

Movie 2. EEG source amplitudes for high $\beta(24-30 \mathrm{~Hz})$ frequencies during the fast movement cadence as function of the phase in a movement cycle. Phase axis in radian, ranging from $-\pi$ to $\pi$, is centered $(0)$ at the maximal flexion of the fingers. Relative amplitude decreases/increases are illustrated in blue/red. EEG source am plitude threshold was set to $50 \%$ of the color scale maximum; the minimum cluster size was set to 20 vertices. and auditory (Fujioka et al., 2012) stimuli in absence of actual movements. In this study, we investigated the opposite scenario, where no external stimuli were provided during rhythmic movement execution. Naturally, sensory inputs of the fingers are present during movements. Consequently, $\beta$ MPA we report may represent processes related to the prediction and integration of this information. Saleh et al. (2010) observed $\beta$ synchrony in the primary motor cortex only for behaviorally relevant, anticipated cues that required attention. Indeed, high $\beta$ synchronization in prefrontal areas was found to be involved in top-down attention and control (Buschman and Miller, 2007; Buschman et al., 2012). Therefore, we interpret the prefrontal MPA cluster we found for $\beta 2$ frequencies (Fig. 6b) to reflect additional top-down control during the faster movement cadence. Moreover, analyzing amplitude comodulation between cortical areas showed that prefrontal $\beta 2$ MPA precedes those in the right sensorimotor seed (Fig. 7). This relationship is also visible in Movie 2 where $\beta 2$ MPAs travel from frontal toward sensorimotor and parietal areas, contributing to the modulation in these regions. For the slow movement cadence, we found two $\beta$ peaks in a movement cycle in sensorimotor regions (Fig. 3f). Based on Movie 1, we suggest that these two peaks are related to two different processing streams. The first involves sensorimotor processing in bilateral hand regions; the second one is more related to central sensorimotor regions and SMA. In contrast to prefrontal $\beta 2$ MPA during fast movements, subcortical regions are leading sensorimotor seed cluster modulations during the slow movement cadence. This may be explained by the basal ganglia and thalamus being involved in top-down control during simple tasks but prefrontal regions during more complex behaviors (Buschman and Miller, 2014). However, precise localization of deep sources based on EEG recordings is challenging. More work is needed to further disentangle $\beta$ MPA and investigate their specific functions. We observed this $\beta$ modulation pattern simultaneously with the much stronger sustained $\beta$ desynchronization we suggest to represent another network. Therefore, the MPA cannot be explained by postmovement $\beta$ synchronization effects. Further, in contrast to the bilateral $\beta$ MPA we report in this work, postmovement $\beta$ synchronization was localized focally to contralateral areas (for review, see Neuper and Pfurtscheller, 2001; Cheyne, 2013).

We also found significant MPA at $\alpha$ and high $\gamma$ frequencies. Their time courses resembled the $\beta$ MPA in sensorimotor areas, but they were temporally shifted in the movement cycle and showed different spatial source patterns. $\alpha$ temporally lagged $\beta 2 \mathrm{MPA}$ in sensorimotor areas. We localized $\alpha$ MPA to bilateral sensorimotor, SMA, and parietal areas, which were stronger in the right hemisphere. Alpha oscillations in these cortical areas are related to working memory during retention of previously shown items (Jensen et al., 2002). Because our paradigm included remembering the movement speed instruction, it would be plausible that the $\alpha \mathrm{MPA}$ we found here represents short-term working memory processes.

We found high $\gamma \mathrm{MPA}$ in the contralateral hand region, which is in agreement with invasive studies showing a close relation of high $\gamma$ amplitudes to movement sequences (Miller et al., 2009; Hermes et al., 2012). An interesting finding is that only the SMA cluster reached significance during the faster movement cadence (Fig. 6b). In these clusters, high $\gamma$ amplitudes were conversely modulated to the high $\beta$ MPA. This relationship could be explained by the opposing roles of $\beta$ and high $\gamma$ oscillations we discussed above. Moreover, we similarly found amplitudes in the high $\beta /$ low $\gamma$ range to be conversely modulated to high $\gamma$ amplitudes in a gait cycle during walking in humans (Seeber et al., 2015). Further, we previously reported sustained $\alpha$ and $\beta$ ERD along with high $\gamma$ increase during walking (Wagner et al., 2012; Seeber et al., 2014, 2015). Together, these findings suggest 
shared electrophysiological principles of rhythmic finger tapping and walking.

In conclusion, we showed two different types of networks during rhythmic finger movements in this work. First, sustained $\alpha$ and $\beta$ desynchronization may be associated with release of inhibition during movement. Together with high $\gamma$ enhancement, these networks may upregulate excitability in regions specific to the body parts that are prepared to be, or actually are, moved (Pfurtscheller and Lopes da Silva, 1999; Miller et al., 2007). Second, movement phase-related networks, which modulate their synchrony in relation to the flexion and extension sequence of the fingers. We suggest frequency-specific MPA to signify distinct large-scale networks associated with specific functions, including top-down control, sensorimotor prediction, and integration. Our findings suggest that EEG source amplitudes reconstructed in a cortical patch are the summation of simultaneous present overlapping networks. Separating these two types of behavior-related large scale networks we distinguished in this work improves the interpretability of EEG sources and advances to relate them to human motor behavior.

\section{References}

Baillet S, Mosher JC, Leahy RM (2001) Electromagnetic brain mapping. IEEE Signal Processing Magazine 18:14-30. CrossRef

Brookes MJ, Woolrich M, Luckhoo H, Price D, Hale JR, Stephenson MC, Barnes GR, Smith SM, Morris PG (2011) Investigating the electrophysiological basis of resting state networks using magnetoencephalography. Proc Natl Acad Sci U S A 108:16783-16788. CrossRef Medline

Buschman TJ, Miller EK (2007) Top-down versus bottom-up control of attention in the prefrontal and posterior parietal cortices. Science 315: 1860-1862. CrossRef Medline

Buschman TJ, Miller EK (2014) Goal-direction and top-down control. Philos Trans R Soc Lond B Biol Sci. 369:20130471. CrossRef Medline

Buschman TJ, Denovellis EL, Diogo C, Bullock D, Miller EK (2012) Synchronous oscillatory neural ensembles for rules in the prefrontal cortex. Neuron 76:838-846. CrossRef Medline

Buzsáki, G (2006) Rhythms of the brain. Oxford: Oxford UP

Buzsáki G, Anastassiou CA, Koch C (2012) The origin of extracellular fields and currents: EEG, ECoG, LFP and spikes. Nat Rev Neurosci 13:407-420. CrossRef Medline

Cheyne DO (2013) MEG studies of sensorimotor rhythms: a review. Exp Neurol 245:27-39. CrossRef Medline

Crone NE, Miglioretti DL, Gordon B, Sieracki JM, Wilson MT, Uematsu S, Lesser RP (1998a) Functional mapping of human sensorimotor cortex with electrocorticographic spectral analysis: I. Alpha and beta eventrelated desynchronization. Brain 121:2271-2299. CrossRef Medline

Crone NE, Miglioretti DL, Gordon B, Lesser RP (1998b) Functional mapping of human sensorimotor cortex with electrocorticographic spectral analysis: II. Event-related synchronization in the gamma band. Brain 121: 2301-2315. CrossRef Medline

Dale AM, Fischl B, Sereno MI (1999) Cortical surface-based analysis: I. Segmentation and surface reconstruction. Neuroimage 9:179-194. CrossRef Medline

Darvas F, Pantazis D, Kucukaltun-Yildirim E, Leahy RM (2004) Mapping human brain function with MEG and EEG: methods and validation. Neuroimage 23:289-299. CrossRef Medline

Donner TH, Siegel M (2011) A framework for local cortical oscillation patterns. Trends Cogn Sci 15:191-199. CrossRef Medline

Elul R (1971) The genesis of the EEG. Int Rev Neurobiol 15:227-272. Medline

Engel AK, Fries P (2010) Beta-band oscillations-signalling the status quo? Curr Opin Neurobiol 20:156-165. CrossRef Medline

Engel AK, Gerloff C, Hilgetag CC, Nolte G (2013) Intrinsic coupling modes: multiscale interactions in ongoing brain activity. Neuron 80:867-886. CrossRef Medline

Fischl B (2012) FreeSurfer. Neuroimage 62:774-781. CrossRef Medline

Fischl B, Sereno MI, Tootell RB, Dale AM (1999) High-resolution intersubject averaging and a coordinate system for the cortical surface. Hum Brain Mapp 8:272-284. CrossRef Medline
Fujioka T, Trainor LJ, Large EW, Ross B (2012) Internalized timing of isochronous sounds is represented in neuromagnetic $\beta$ oscillations. J Neurosci 32:1791-1802. CrossRef Medline

Gerloff C, Richard J, Hadley J, Schulman AE, Honda M, Hallett M (1998) Functional coupling and regional activation of human cortical motor areas during simple, internally paced and externally paced finger movements. Brain 121:1513-1531. CrossRef Medline

Gramfort A, Papadopoulo T, Olivi E, Clerc M (2010) OpenMEEG: opensource software for quasistatic bioelectromagnetics. Biomed Eng Online 9:45. CrossRef Medline

Hämäläinen MS, Ilmoniemi RJ (1994) Interpreting magnetic fields of the brain: minimum norm estimates. Med Biol Eng Comput 32:35-42. CrossRef Medline

Hermes D, Siero JC, Aarnoutse EJ, Leijten FS, Petridou N, Ramsey NF (2012) Dissociation between neuronal activity in sensorimotor cortex and hand movement revealed as a function of movement rate. J Neurosci 32:97369744. CrossRef Medline

Houweling S, Beek PJ, Daffertshofer A (2010) Spectral changes of interhemispheric crosstalk during movement instabilities. Cereb Cortex 20: 2605-2613. CrossRef Medline

Hyvärinen A (1999) Fast and robust fixed-point algorithms for independent component analysis. IEEE Trans Neural Netw 10:626-634. CrossRef Medline

Jasper HH, Penfield W (1949) Electrocorticograms in man: effect of the voluntary movement upon the electrical activity of the precentral gyrus. Arch Psychiatry Z Neurol 183:163-174. CrossRef

Jenkinson N, Brown P (2011) New insights into the relationship between dopamine, beta oscillations and motor function. Trends Neurosci 34: 611-618. CrossRef Medline

Jensen O, Gelfand J, Kounios J, Lisman JE (2002) Oscillations in the alpha band $(9-12 \mathrm{~Hz})$ increase with memory load during retention in a shortterm memory task. Cereb Cortex 12:877-882. CrossRef Medline

Jolliffe I (2002) Principal component analysis. Encyclopedia of statistics in behavioral science. New York: Wiley.

Joundi RA, Jenkinson N, Brittain JS, Aziz TZ, Brown P (2012) Driving oscillatory activity in the human cortex enhances motor performance. Curr Biol 22:403-407. CrossRef Medline

Kybic J, Clerc M, Abboud T, Faugeras O, Keriven R, Papadopoulo T (2005) A common formalism for the integral formulations of the forward EEG problem. IEEE Trans Med Imaging 24:12-28. CrossRef Medline

Maris E, Oostenveld R (2007) Nonparametric statistical testing of EEG and MEG data. J Neurosci Methods 164:177-190. CrossRef Medline

Michel CM, Murray MM (2012) Towards the utilization of EEG as a brain imaging tool. Neuroimage 61:371-385. CrossRef Medline

Michel CM, Murray MM, Lantz G, Gonzalez S, Spinelli L, Grave de Peralta R (2004) EEG source imaging. Clin Neurophysiol 115:2195-2222. CrossRef Medline

Miller KJ, Leuthardt EC, Schalk G, Rao RP, Anderson NR, Moran DW, Miller JW, Ojemann JG (2007) Spectral changes in cortical surface potentials during motor movement. J Neurosci 27:2424-2432. CrossRef Medline

Miller KJ, Zanos S, Fetz EE, den Nijs M, Ojemann JG (2009) Decoupling the cortical power spectrum reveals real-time representation of individual finger movements in humans. J Neurosci 29:3132-3137. CrossRef Medline

Morlet J, Arens G, Fourgenau E, Glard D (1982) Wave propagation and sampling theory: I. Complex signal and scattering in multilayered media. Geophysics 47:222-236. CrossRef

Müller GR, Neuper C, Rupp R, Keinrath C, Gerner HJ, Pfurtscheller G (2003) Event-related beta EEG changes during wrist movements induced by functional electrical stimulation of forearm muscles in man. Neurosci Lett 340:143-147. CrossRef Medline

Neuper C, Pfurtscheller G (2001) Event-related dynamics of cortical rhythms: frequency-specific features and functional correlates. Int J Psychophysiol 43:41-58. CrossRef Medline

Nichols TE, Holmes AP (2002) Nonparametric permutation tests for functional neuroimaging: a primer with examples. Hum Brain Mapp 15:1-25. Medline

Nolte G, Bai O, Wheaton L, Mari Z, Vorbach S, Hallett M (2004) Identifying true brain interaction from EEG data using the imaginary part of coherency. Clin Neurophysiol 115:2292-2307. CrossRef Medline

Oostenveld R, Praamstra P (2001) The five percent electrode system for high-resolution EEG and ERP measurements. Clin Neurophysiol 112: 713-719. 
Pascual-Marqui RD (2002) Standardized low resolution brain electromagnetic tomography (sLORETA): technical details. Methods Findings Exp Clin Pharmacol 24D:5-12. Medline

Pfurtscheller G, Aranibar A (1977) Event-related cortical desynchronization detected by power measurements of scalp EEG. Electroencephalogr Clin Neurophysiol 42:817-826. CrossRef Medline

Pfurtscheller G, Lopes da Silva FH (1999) Event-related EEG/MEG synchronization and desynchronization: basic principles. Clin Neurophysiol 110:1842-1857. CrossRef Medline

Pfurtscheller G, Neuper C, Andrew C, Edlinger G (1997) Foot and hand area mu rhythms. Int J Psychophysiol 26:121-135. CrossRef Medline

Pogosyan A, Gaynor LD, Eusebio A, Brown P (2009) Boosting cortical activity at beta-band frequencies slows movement in humans. Curr Biol 19:1637-1641. CrossRef Medline

Pollok B, Gross J, Müller K, Aschersleben G, Schnitzler A (2005) The cerebral oscillatory network associated with auditorily paced finger movements. Neuroimage 24:646-655. CrossRef Medline

Raichle ME (2010) Two views of brain function. Trends Cogn Sci 14:180190. CrossRef Medline

Saleh M, Reimer J, Penn R, Ojakangas CL, Hatsopoulos NG (2010) Fast and slow oscillations in human primary motor cortex predict oncoming behaviorally relevant cues. Neuron 65:461-471. CrossRef Medline

Scherer R, Zanos SP, Miller KJ, Rao RP, Ojemann JG (2009) Classification of contralateral and ipsilateral finger movements for electrocorticographic brain-computer interfaces. Neurosurg Focus 27:E12. CrossRef Medline
Seeber M, Scherer R, Wagner J, Solis-Escalante T, Müller-Putz GR (2014) EEG beta suppression and low gamma modulation are different elements of human upright walking. Front Hum Neurosci 8:485. CrossRef Medline

Seeber M, Scherer R, Wagner J, Solis-Escalante T, Müller-Putz GR (2015) High and low gamma EEG oscillations in central sensorimotor areas are conversely modulated during the human gait cycle. Neuroimage 112: 318-326. CrossRef Medline

Siegel M, Donner TH, Engel AK (2012) Spectral fingerprints of large-scale neuronal interactions. Nat Rev Neurosci 13:121-134. CrossRef Medline

Tadel F, Baillet S, Mosher JC, Pantazis D, Leahy RM (2011) Brainstorm: a user-friendly application for MEG/EEG analysis. Comput Intelligence Neurosci 2011.

Toma K, Mima T, Matsuoka T, Gerloff C, Ohnishi T, Koshy B, Andres F, Hallett M (2002) Movement rate effect on activation and functional coupling of motor cortical areas. J Neurophysiol 88:3377-3385. CrossRef Medline

Wagner J, Solis-Escalante T, Grieshofer P, Neuper C, Müller-Putz G, Scherer R (2012) Level of participation in robotic-assisted treadmill walking modulates midline sensorimotor EEG rhythms in able-bodied subjects. Neuroimage 63:1203-1211. CrossRef Medline

Wagner J, Solis-Escalante T, Scherer R, Neuper C, Müller-Putz G (2014) It's how you get there: walking down a virtual alley activates premotor and parietal areas. Front Hum Neurosci 8:93. CrossRef Medline

Yuan H, Perdoni C, He B (2010) Relationship between speed and EEG activity during imagined and executed hand movements. J Neural Eng 7:26001. CrossRef Medline 